\title{
Aging, proteotoxicity, mitochondria, glycation, NAD+ and carnosine: possible inter-relationships and resolution of the oxygen paradox
}

\author{
Alan R. Hipkiss* \\ School of Clinical and Experimental Medicine, College of Medical and Dental Sciences, The University of Birmingham, Birmingham, UK
}

\author{
Edited by: \\ Merce Pallas, University of Barcelona, \\ Spain \\ Reviewed by: \\ Ana M. Coto-Montes, \\ University of Oviedo, Spain \\ Daniel Ortuño-Sahagun, \\ Centro Universitario de Ciencias \\ Biológicas y Agropecuarias, Mexico \\ Merce Pallas, University of Barcelona, \\ Spain \\ *Correspondence: \\ Alan R. Hipkiss, School of Clinical and \\ Experimental Medicine, College of \\ Medical and Dental Sciences, The \\ University of Birmingham, Edgbaston, \\ Birmingham B15 2TT, UK. \\ e-mail: alanandjill@lineone.net
}

It is suggested that $\mathrm{NAD}^{+}$availability strongly affects cellular aging and organism lifespan: low $\mathrm{NAD}^{+}$availability increases intracellular levels of glycolytic triose phosphates (glyceraldehyde3-phosphate and dihydroxyacetone-phosphate) which, if not further metabolized, decompose spontaneously into methylglyoxal (MG), a glycating agent and source of protein and mitochondrial dysfunction and reactive oxygen species (ROS). MG-damaged proteins and other aberrant polypeptides can induce ROS generation, promote mitochondrial dysfunction and inhibit proteasomal activity. Upregulation of mitogenesis and mitochondrial activity by increased aerobic exercise, or dietary manipulation, helps to maintain $\mathrm{NAD}^{+}$availability and thereby decreases MG-induced proteotoxicity. These proposals can explain the apparent paradox whereby aging is seemingly caused by increased ROS-mediated macromolecular damage but is ameliorated by increased aerobic activity. It is also suggested that increasing mitochondrial activity decreases ROS generation, while excess numbers of inactive mitochondria are deleterious due to increased ROS generation. The muscle- and brain-associated dipeptide, carnosine, is an intracellular buffer which can delay senescence in cultured human fibroblasts and delay aging in senescenceaccelerated mice. Carnosine's ability to react with MG and possibly other deleterious carbonyl compounds, and scavenge various ROS, may account for its protective ability towards ischemia and ageing.

Keywords: ageing, methylglyoxal, ROS, exercise, proteolysis, mitogenesis, glycolysis

\section{INCREASED MITOCHONDRIAL DYSFUNCTION AND ALTERED PROTEIN ACCUMULATION - ASSOCIATION WITH AGING}

Two strong correlates of aging are mitochondrial dysfunction (Sastre et al., 2003; Sedensky and Morgan, 2006; Figueiredo et al., 2008; Wei et al., 2009) and accumulation of altered proteins (Hipkiss, 2006a; Soskic et al., 2007; Lindner and Demarez, 2009). It is possible that both these apparent effects of aging may also be causal to aging and much age-related pathology. Although many experiments have shown that mitochondrial dysfunction frequently accompanies aging, whether aging causes mitochondrial dysfunction, or whether mitochondrial dysfunction causes aging, is debated. For example, while aging is accompanied by the accumulation of altered proteins, possibly due to a decreased ability of the intracellular proteases (lysosomal and proteasomal) to degrade aberrant polypeptide chains (Carrard et al., 2002; Bergamini et al., 2007; Chondrogianni and Gonos, 2007; Hanson et al., 2008; Kurz et al., 2008a; Yun et al., 2008), it has also been shown that altered proteins can bind to mitochondria and compromise their function (Hansson Petersen et al., 2008; Devi and Anandatheerthavarada, 2009; Rhein et al., 2009; Sun et al., 2009). Furthermore there is strong evidence for the proposal that decreased autophagy and lysosomal activity compromises elimination of dysfunctional mitochondria (Brunk and Terman, 2002; Terman et al., 2003; Kim et al., 2007; Kurz et al., 2008b), thereby promoting their accumulation. Conversely, studies in nematode Caenorhabditis elegans (Hanson et al., 2008; Toth et al.,
2008), Drosophila (Simonsen et al., 2008) and yeast (Alvers et al., 2009) have shown that lifespan in these organisms was extended when autophagy was upregulated, which could improve cellular ability to eliminate dysfunctional mitochondria (i.e. stimulate mitophagy). Alternatively/additionally, mitochondrial dysfunction increases generation of oxygen free-radicals and related reactive oxygen species (collectively termed ROS) which in turn damage proteins to such an extent that cross-linked polypeptides are generated which not only resist proteolytic attack, but also inhibit proteasomal activity, thereby establishing a deleterious cycle which would be likely to eventually result in the cell's demise. However, when lifespan was extended by calorie restriction, proteolysis (autophagic and proteasomal) was found to be stimulated, which again could help to eliminate dysfunctional organelles and altered proteins (Cuervo, 2008). It is also possible that excessive autophagy may be deleterious as increased autophagic activity accompanies aging in progeroid mice (Marino and Lopez-Otin, 2008). Additionally that the $\beta$-amyloid peptide (1-42), commonly associated with Alzheimer's disease, can bind to mitochondrial complex-1 NADH dehydrogenase (Munguia et al., 2005), while deficiency in fatty acid beta-oxidation pathway may also be associated with neurodegeneration, possibly due to binding of $\beta$-amyloid peptide to hydroxyacyl-CoA dehydrogenase (Oppermann et al., 1999), further illustrate the possible relationships between altered proteins, mitochondrial dysfunction and age-related pathology. 


\section{AGING, NAD ${ }^{+}$AND METHYLGLYOXAL}

Whatever the cause of mitochondrial dysfunction, one consequence of lowered mitochondrial activity will be decreased mitochondriallymediated regeneration of $\mathrm{NAD}^{+}$from NADH. Oxidation of the glycolytic intermediate glyceraldehyde-3-phosphate (G3P) by glyceraldehyde-3-phosphate dehydrogenase requires $\mathrm{NAD}^{+}$ (which is converted to NADH). As a continuous supply of $\mathrm{NAD}^{+}$ is required for triose phosphate metabolism to procede, both G3P and dihydroxyacetone-phosphate (DHAP) will accumulate should $\mathrm{NAD}^{+}$availability be restricted. Accumulation of these trioses is deleterious because both can spontaneously decompose into methylglyoxal (MG) (Turk, 2009), a highly toxic glycating agent which readily damages proteins by reacting with amino and guanidino groups of lysine and arginine residues, respectively (Rabbani and Thornalley, 2008a). Indeed, a number of experiments have demonstrated the damaging effects of MG on mitochondrial activity (Johans et al., 2005; SinhaRoy et al., 2005; Rabbani and Thornalley, 2008b; Wang et al., 2009), including increased ROS formation in mitochondria as well as in the cytoplasm (Desai and Wu, 2008). Increased MG formation is also a likely explanation for many molecular events accompanying cell senescence (Sejersen and Rattan, 2009), ischemia, hyperglycemia and associated pathology (Nicolay et al., 2006; Rabbani and Thornalley, 2008a). Significantly, experiments in C. elegans have shown that increased expression of the MG detoxification enzyme, glyoxalase-1, increases organism lifespan, most likely by lowering MG levels and thereby decreasing MG-mediated protein damage and mitochondrial dysfunction (Morcos et al., 2008; Schlotterer et al., 2009). Furthermore, increasing dietary intake of MG-glycated protein can eliminate the beneficial effects on mouse lifespan induced by dietary restriction (Cai et al., 2007). As everyother-day feeding without any overall reduction in calorie intake can also increase organism lifespan (Masternak et al., 2005; Mattson and Wan, 2005; Martin et al., 2006), it is possible that periods of fasting, during which MG formation from glycolytic trioses will be suppressed, may provide an explanation for the observed beneficial effects of dietary restriction on organism lifespan (Hipkiss, 2006b, 2008). Collectively, these observations suggest that factors which regulate either MG formation or its elimination can exert critical roles in aging (Hipkiss, 2009a).

\section{MECHANISMS CONTROLLING MG-MEDIATED MACROMOLECULAR DAMAGE}

As noted above, a major influence on intracellular MG formation from the glycolytic trioses G3P and DHAP is the availability of $\mathrm{NAD}^{+}$. Mitochondria provide a major aerobic route for the regeneration of $\mathrm{NAD}^{+}$from $\mathrm{NADH}$ which occurs via the operation of the oxaloacetate-malate shuttle and the glycerol-phosphate cycle. An anaerobic mechanism whereby $\mathrm{NAD}^{+}$is regenerated from $\mathrm{NADH}$ is carried out in yeast by the reduction of acetaldehyde to ethyl alcohol, whereas in animal tissues, due to the absence of the enzyme pyruvate decarboxylase, the conversion of pyruvic acid to lactic acid is the analogous reaction. The latter process, however, imposes the additional metabolic stress of acidosis by increasing the number of hydrogen ions in the cytoplasm. In tissues where this process is particularly important, such as fast-twitch glycolytic muscle, additional buffering capacity is provided by the dipeptide carnosine ( $\beta$-alanyl-L-histidine) and related imidazole dipeptides
(Abe, 2000). Interestingly, it appears that carnosine, which is also found in brain, especially the olfactory lobe, can react with MG and other metabolic aldehydes and may be generally protective against aldehyde-mediated macromolecular damage (Hipkiss, 2009b,c). Other experiments have demonstrated that carnosine can suppress the deleterious effects of ischemia in brain (Dobrota et al., 2005), liver (Fouad et al., 2007) and kidney (Fujii et al., 2005), possibly mediated by the dipeptide's reaction with MG (Hipkiss and Chana, 1998; Aldini et al., 2005) generated as a consequence of hypoxiainduced failure to regenerate $\mathrm{NAD}^{+}$from NADH. These antiischemic effects of the dipeptide are consistent with the proposal that, by reacting directly with MG, carnosine suppresses dicarbonyl toxicity. That ischemia-related ROS formation and consequent proteotoxicity are prevented by upregulation of glyoxalase- 1 in $C$. elegans (Morcos et al., 2008; Schlotterer et al., 2009), reinforces the idea that raised MG levels are significant in ischemia. The observation that defects in triose phosphate isomerase activity, which promotes DHAP accumulation, induces many of the deleterious effects associated with hyperglycemia and aging (Orosz et al., 2009), further supports the proposal that MG plays a causal role in much age-related proteotoxicity (Hipkiss, 2008, 2009a). Significantly, experiments have shown that carnosine delays aging in cultured human fibroblasts (McFarland and Holliday, 1994) and senescenceaccelerated mice (Yuneva et al., 1999, 2002). The fact that carnosine has also been reported to (i) possess anti-oxidant activity (Kohen et al., 1988; Bogardus and Boissonneault, 2000; Calabrese et al., 2005), (ii) induce stress-protein expression (when complexed with zinc ions) (Odashima et al., 2006; Ohkawara et al., 2006; Wada et al., 2006) and (iii) decrease telomere shortening (Shao et al., 2004), reinforces the idea that this essentially non-toxic dipeptide is supportive of longevity (Hipkiss, 2009b,c).

\section{NAD+ AND PROTEOTOXICITY}

The observations outlined above reinforce the likely importance, to aging and related disorders, of MG, whose generation is affected by $\mathrm{NAD}^{+}$availability. Additionally, $\mathrm{NAD}^{+}$is important for stress-protein synthesis (Westerheide et al., 2009), autophagic activity (Lee et al., 2008; Salminen and Kaamiranta, 2009), sirtuin-mediated protein deacetylation (Bordone and Guarente, 2005; Rodgers et al., 2008) and increased mitogenesis (Bonawitz et al., 2007; Cunningham et al., 2007), all of which impact upon the processes influencing proteostasis and aging. Thus the beneficial effects of this virtuous cycle could help suppress the onset of cellular aging by decreasing the potential for MG generation, as well as improving protein quality control. It follows that putative pluripotent protective agents such as carnosine could also help suppress the deleterious effects of age-related disorders such as Alzheimer's disease (Hipkiss, 2007) and type-2 diabetes (Hipkiss, 1998,2009 d), both of which seem to possess a number of overlapping phenotypic characteristics, including increased protein glycoxidation (Maher and Schubert, 2009).

\section{IF ROS ARE CAUSAL TO AGING, WHY IS AGING SUPPRESSED BY INCREASED AEROBIC ACTIVITY? A PARADOX RESOLVED?}

Despite the detection of increased levels of oxidatively-damaged macromolecules in aging cells and tissues, it is surely paradoxical that aging and much related pathology can be delayed by 
increased mitochondrial activity and aerobic exercise. It is possible to resolve this paradox however. It is suggested that mitochondria, by ensuring $\mathrm{NAD}^{+}$regeneration from $\mathrm{NADH}$, suppress $\mathrm{MG}$ formation (and hence its deleterious effects) and thereby partly explains the beneficial effects of increased aerobic activity towards much age-related dysfunction. Lowered MG generation may also result from the increased synthesis of nucleic acid precursors. This would divert glucose metabolism via the pentose phosphate pathway, which would not only increase glutathione synthesis via increased NADPH generation, but also decrease synthesis of MG precursors, triose phosphates. Furthermore the upregulation in synthesis of mitochondrial proteins may also be accompanied by increased synthesis of the necessary proteases and chaperone proteins to help maintain protein quality (Hipkiss, 2009e). However, it should be pointed out that ROS may also provide a necessary signal for increased mitogenesis in exercised muscle.

It has been shown that limb immobilisation promotes an increase in mitochondrial ROS generation (Pesce et al., 2002; Miller et al., 2007; Bar-Shai et al., 2008), observations consistent with the suggestion that inactive mitochondria generate more ROS than those actively respiring (Van Voorhies, 2004; Barja, 2007; Burhans and Weinberger, 2007; Brooks et al., 2008; Holloszy, 2008; Hood, 2009). Experiments have demonstrated that uncoupling electron transport from ATP synthesis suppresses aging and can extend lifespan (Speakman et al., 2004; Wolkow and Iser, 2006; Hood, 2009); this would increase $\mathrm{NAD}^{+}$generation from NADH (Liu et al., 2008) and thereby decrease the potential for MG synthesis. Resveratrol and rapamycin, both of which have been shown to exert anti-aging effects, promote mitochondrial activity and mitogenesis, whilst also seeming to suppress glycolysis (Knutson and Leeuwenburgh, 2008; Orallo, 2008; Cox and Mattison, 2009; Harrison et al., 2009) thereby decreasing the potential for MG synthesis. There is also considerable evidence showing that acetyl-L-carnitine, which facilitates entry of fatty acid acyl-units into mitochondria thereby increasing mitochondrial activity, has anti-aging effects and decreases ROS generation (Mollica et al., 2001; Liu et al., 2002; Virmani et al., 2002; Poon et al., 2006). It should also be noted, however, that it is still uncertain whether all age-related increases in ROS formation is intramitochondrial in origin; ROS can be generated outside mitochondria e.g. by MG, glycated proteins and cytoplasmic $\mathrm{NAD}(\mathrm{P}) \mathrm{H}$ oxidase and xanthine oxidase activities.

\section{ARE TOO MANY MITOCHONDRIA DELETERIOUS?}

The above proposal suggests that increased mitochondria activity, and in particular mitogenesis, may be considered as anti-aging processes. It is also possible that the presence of excess inactive mitochondria (i.e. those not carrying out electron transport) are actually deleterious. If oxygenated mitochondria are supplied with insufficient electrons, for example when ATP demand is low, this will increase the potential for creation of incompletely reduced oxygen atoms i.e. superoxide $\mathrm{O}_{2}^{-}$, an oxygen free-radical. As mentioned above, limb immobilization results in increased ROS generation (Pesce et al., 2002; Miller et al., 2007; Bar-Shai et al., 2008) which may be an example of such a phenomenon. Furthermore increased mitochondrial degradation, which occurs in response to decreased muscle activity in animal and human studies (Hood, 2009), could be a metabolic response to the presence of excess inactive mitochondria, perhaps stimulated by increased ROS generation in inactive organelles. Indeed ROS-mediated protein modification increases proteolytic susceptibility, both intramitochondrially and in the cytosol, creates a precedent for ROS-induced damage increasing the potential for catabolic attack. These observations can be interpreted as suggesting that excess inactive mitochondria are indeed deleterious.

Regulation of mitochondrial numbers is undoubtably complex, as illustrated in a recent study in C. elegans which revealed that the protein prohibitin is important in this process (Artal-Sanz and Tavernarakis, 2009). Significantly, the results showed that the beneficial effect of increased mitogenesis (induced by prohibitin deficiency) upon longevity was strongly influenced by organism metabolism. It was found that raised mitochondrial synthesis promoted an increase in organism lifespan at 25 degrees, and at 35 degrees the organisms were also strongly thermotolerant. While at a lower temperature (20 degrees) decreased longevity was observed in prohibitin-deficient nematodes. These complex and seemingly paradoxical findings can be successfully explained if one assumes that inactive mitochondria produce more ROS than mitochondria actively generating ATP (as proposed above). Hence it can be suggested that many mitochondria are inactive at the lower temperature due to low energy demands and consequently ROS formation is increased and longevity compromised. Whereas, the increased ATP demand at the higher temperature results in increased mitochondrial activity, which, correspondingly, decreases ROS generation and increases lifespan. Additionally, however, temperature-dependent differences in oxygen solubility could influence ROS formation and thereby affect the organism longevity; as oxygen solubility is inversely related to temperature, raising the temperature could decrease mitochondrial oxygen supply and which could decrease the amounts of ROS generated.

\section{CONCLUSION}

It is suggested that maintenance of mitochondrial activity helps to suppress aging by ensuring $\mathrm{NAD}^{+}$availability which decreases $M G$ generation from glycolytic trioses and subsequent formation of toxic MG-modified (glycated) proteins. Furthermore, increased mitogenesis will divert glucose metabolites through the pentose phosphate cycle for ribose and deoxyribose synthesis, which additionally will increase glutathione synthesis, via increased NADPH formation. It is likely that lowering the potential for MG formation will be additionally beneficial by helping to maintain proteostasis and delaying proteostatic collapse, because highly glycated (cross-linked) proteins can compromise protein quality control by inhibiting proteasome activity. Furthermore it is possible that continuous mitogenesis and growth in the young suppress onset of aging phenomena, even when the organism possesses a genetic predisposition for age-related pathology. However, once growth ceases, the potential for MG formation increases, with the inevitable finitude of life pressaged by proteostatic collapse in which altered proteins damage mitochondria, induce ROS formation which then damage more proteins, eventually promoting apoptosis. This would suggest that any process (e.g. increased ROS generation, increased protein glycation, decreased altered protein elimination, increased synthesis of erroneous proteins etc.) that causes intracellular accumulation of altered proteins could provide 
the initiating causal event which promotes aging onset. Whilst increased mitogenesis may be beneficial in some circumstances, it is also suggested that excess numbers of inactive mitochondria could be an additional source of ROS and, by overwhelming cytosolic anti-oxidant activity, have adverse effects on stress resistance, cellular viability and organism longevity.

\section{REFERENCES}

Abe, H. (2000). Role of histidine-related compounds as intracellular proton buffering constituents. Biochemistry (Moscow) 65, 757-765.

Aldini, G., Maffei-Fracino, R., Beretta, G., and Carini, M. (2005). Carnosine and related dipeptides as quenchers of reactive carbonyl species: from structural studies to therapeutic perspectives. Biofactors 24, 77-87.

Alvers, A. L., Fishwick, L. K., Wood, M. S., Hu, D., Chung, H. S., Dunn, W. A., and Aris, J. P. (2009). Autophagy and amino acid homeostasis are required for chronological longevity in Saccharomyces cerevisiae. Aging Cell 8, 353-369.

Artal-Sanz, M., and Tavernarakis, N. (2009). Prohibitin couples diapause signalling to mitochondrial metabolism during ageing in C. elegans. Nature 461, 793-797.

Barja, G. (2007). Mitochondrial oxygen consumption and reactive oxygen species production are independently modulated: implications for aging studies. Rejuvenation Res. 10, 215-224.

Bar-Shai, M., Carmeli, E., Ljubuncic, P., and Reznick, A.Z. (2008). Exercise and immobilization in aging animals: the involvement of oxidative stress and NF-kappaB activation. Free Radic. Biol. Med. 44, 202-214.

Bergamini, E., Cavallini, G., Donati, A., and Gori, Z. (2007). The role of autophagy in aging. Its essential part in the anti-aging mechanism of caloric restriction. Ann. N. Y. Acad. Sci. 1114, 69-78.

Bogardus, S. L., and Boissonneault, G. A. (2000). Carnosine inhibits in vitro low-density lipoprotein oxidation. Nutr. Res. 20, 967-976.

Bonawitz, N. D., Chatenay-Lapointe, M., Pan, Y., and Shadel, G. S. (2007). Reduced TOR signalling extends chronological life span via increased respiration and upregulated mitochondrial gene expression. Cell Metab. 5, 265-277.

Bordone, L., and Guarente, L. (2005). Calorie restriction, sirtl and metabolism: understanding longevity. Nat. Rev. Mol. Cell Biol. 6, 298-305.

Brooks, S. V., Vasilaki, A., Larkin, L. M., McArdle, A., and Jackson, M. J. (2008). Repeated bouts of aerobic exercise lead to reductions in skeletal muscle free radical generation and nuclear factor kappa $\beta$ activation. J. Physiol. 586, 3979-3990.
Brunk, U. T., and Terman, A. (2002). The mitochondrial-lysosomal axis theory of aging. Accumulation of damaged mitochondria as a result of imperfect autophagocytosis. Eur. J. Biochem. 269, 1996-2002.

Burhans, W. C., and Weinberger, M. (2007). DNA replication stress, genome instability and aging. Nucleic Acid Res. 35, 7545-7556.

Cai, W., He, J. C., Zhu, L., Chen, X., Wallenstein, S., Striker, G. E., and Vlassara, H. (2007). Reduced oxidant stress and extended lifespan in mice exposed to a low glycotoxin diet. Association with increased AGER1 expression. Am. J. Pathol. 170, 1893-1902.

Calabrese, V., Coombrita, C., Guagliano, E., Sapoenza, M., Ravagna, A., Cardile, V., Scapagnini, G., Santoro, A. M., Mangiameli, A., Butterfield, D. A., Giuffirda Stella, A. M., and Rizzarelli, E. (2005). Protective effect of carnosine during nitrosactive stress in astroglial cell cultures. Neurochem. Res. 30, 797-807.

Carrard, G., Bulteau, A.-L., Petropoulos, I., and Friguet, B. (2002). Impairment of proteasome structure and function in aging. Int. J. Biochem. Cell Biol. 34, 1461-1474.

Chondrogianni, N., and Gonos, E. S. (2007). Overexpression of hUMP11/ POMP proteasome accessory protein enhances proteasome-mediated antioxidant defence. Exp. Gerontol. 42, 899-903.

Cox, L. S., and Mattison, J. A. (2009). Increased longevity through caloric restriction or rapamycin feeding in mammals: common mechanisms for common outcomes? Aging Cell 8, 607-613.

Cuervo, A. M. (2008). Calorie restriction and aging: the ultimate "cleansing diet.” J. Gerontol. Biol. Sci. 63A, 547-549.

Cunningham, J. T., Rodgers, J. T., Arlow, D. H., Vazquez, F., Mootha, V. K., and Puigserver, P. (2007). mTOR controls mitochondrial oxidative function through a YY1-PGC-1 $\alpha$ transcription complex. Nature 450, 736-740.

Desai, K. M., and Wu, L. (2008). Free radical generation by methylglyoxal in tissues. Drug Metab. Drug Interact. 23, 151-173.

Devi, L., and Anandatheerthavarada, H. K. (2009). Mitochondrial trafficking of APP and alpha-synuclein: relevance to mitochondrial dysfunction in Alzheimer's and Parkinson's diseases. Biochim. Biophys. Acta (in press) PMID 19619643.

Dobrota, D., Fedorova, T., Stvolinsky, S. L., Babusikova, E., Likavcanova, K., Drgova, A., Strapkova, A., and Boldyrev, A.A. (2005).Carnosine protects the brain of rats and Mongolian gerbils against ischemic injury: after-stroke-effect. Neurochem. Res. 30, 1283-1288.

Figueiredo, P. A., Mota, M. P., Apell, H. J., and Duarte, J. A. (2008). The role of mitochondria in aging of skeletal muscle. Biogerontology 9, 67-84.

Fouad, A. A., El-Rehany, M. A., and Maghraby, H. K. (2007). The hepatoprotective effect of carnosine against ischemia/reperfusion liver injury in rats. Eur. J. Pharmacol. 572, 61-68.

Fujii, T., Takaoka, M., Tsuruoka, N., Kiso Y., and Matsumura, Y. (2005). Dietary supplemention with L-carnosine prevents ischemia/reperfusion renal injury in rats. Biol. Pharm. Bull. 28, 361-363.

Hanson, M., Chandros, A., Mitic, L. L., Onken B., Driscoll, M., and Kenyon, C. (2008). A role for autophagy in the extension of lifespan by dietary restriction in C. elegans. PLoS Genet. 4, e24 doi: 10.1371/journal.pgen.0040024.

Hansson Petersen, C. A., Alikhani, N., Behbahani, H., Wiehager, B., Pavlov, P. F., Alafuzoff, I., Leinonen, V., Ito, A., Winblad, B., Glaser, E., and Ankarcrona, M. (2008). The amyloid beta-peptide is imported into mitochondria via the TOM import machinery and localized to mitochondrial cristae. Proc. Natl. Acad. Sci. U.S.A. 105, 13145-13150.

Harrison, E., Strong, R., Sharp, Z. D., Nelson, J. F., Astle, C. M., Flurkey, K. Nadon, N. L., Wilkinson,. E., Frenkel, K., Carter, C. S., Pahor, M., Javors, M. A., Fernandez, E., and Miller, R. A. (2009). Rapamycin fed late in life extends the lifespan in genetically heterogeneous mice. Nature 460, 392-395.

Hipkiss, A. R. (1998). Carnosine, a protective anti-ageing peptide? Int. J. Biochem. Cell Biol. 30, 863-868.

Hipkiss, A. R. (2006a). Accumulation of altered proteins and ageing: causes and effects. Exp. Gerontol. 41, 464-473.

Hipkiss, A. R. (2006b). Caloric restriction and ageing-is glycolysis the problem? Mech. Ageing Dev. 127, 8-15.

Hipkiss, A. R. (2007). Could carnosine or related structures suppress Alzheimer's disease? J. Alzheimers Dis. 11, 229-240.
Hipkiss, A. R. (2008). Energy metabolism, altered proteins, siruins and ageing: converging mechanisms? Biogerontology 9, 49-55.

Hipkiss, A. R. (2009a). NAD ${ }^{+}$availability and proteotoxicity. Neuromolecular Med. 11, 97-100.

Hipkiss, A. R. (2009b). On the enigma of carnosine's anti-ageing actions. Exp. Gerontol. 44, 237-242.

Hipkiss, A. R. (2009c). Carnosine and its possible roles in nutrition and health. Adv. Food Nutr. Res. 57, 87-154.

Hipkiss A. R. (2009d). Carnosine, diabetes and Alzheimer's disease. Expert Rev. Neurother. 9, 1-4.

Hipkiss, A. R. (2009e). Error-protein metabolism and ageing. Biogerontology 10, 523-529.

Hipkiss, A. R., and Chana, H. (1998). Carnosine protects proteins against methylglyoxal-mediated modifications. Biochem. Biophys. Res. Commun. 248, 28-32.

Holloszy, J. O. (2008). Regulation by exercise of skeletal muscle content of mitochondria and Glut4. J. Physiol. Pharmacol. 59(Suppl. 7), 5-18.

Hood, D. A. (2009). Mechanisms of exercise-induced mitochondrial biogenesis in skeletal muscle. Appl. Physiol. Nutl. Metab. 34, 465-472.

Johans, M., Milanesi, E., Franck, M., Johans, C., Liobikas, J., Panagiotaki, M., Greci, L., Principato, G., Kinnunen, P. K. J., Bernardi, P., Costantini, P., and Eriksson, O. (2005). Modification of permeability transition pore arginine(s) by phenylglyoxal derivatives in isolated mitochondria and mammalian cells. Structure-function relationship of arginine ligands. J. Biol. Chem. 280, 12130-12136.

Kim, I., Rodriguez-Enriquez, S., and Lemasters, J. J. (2007). Selective degradation of mitochondria by mitography. Arch. Biochem. Biophys. 462, 245-253.

Knutson, M. D., and Leeuwenburgh, C. (2008). Resveratrol and novel potent activiators of SIRT1: effects on aging and age-related diseases. Nutr. Rev. 66, 591-596.

Kohen, R., Yamamoto, Y., Cundy, K. C., and Ames, B. N. (1988). Antioxidant activity of carnosine, homocarnosine and anserine present in muscle and brain. Proc. Natl. Acad. Sci. U.S.A. 95, 2175-2179.

Kurz, T., Terman, A., Gustafsson, B., and Brunk, U. T. (2008a). Lysosomes and oxidative stress in aging and apoptosis. Biochim. Biophys. Acta 1780 , 1291-1303. 
Kurz, T., Terman, A., Gustafasson, B., and Brunk, U. T. (2008b). Lysosomes in iron metabolism, aging and apoptosis. Histochem. Cell Biol. 129, 389-406.

Lee, I. H., Cao, L., Mostoslavsky, R., Lombard, D. B., Liu, J., Bruns, N., Tsokos, M., Alt. F. W., and Kinklel, T. (2008).A role for the NAD-dependent deacetylase Sirt1 in the regulation of autophagy. Proc. Natl. Acad. Sci. U.S.A. 105, 3374-3379.

Lindner, A. B., and Demarez, A. (2009). Protein aggregation as a paradigm of aging. Biochim. Biophys. Acta 1790, 980-996.

Liu, D., Pitts, M., and Mattson, M. P. (2008). Preventing $\mathrm{NAD}^{+}$depletion protects neurons against excitoxicity: bioenergetic effects of mild mitochondrial uncoupling and caloric restriction. Ann. N. Y. Acad. Sci. 1147, 275-282.

Liu, J., Killilea, D. W., and Ames, B. N. (2002). Age-associated mitochondrial oxidative decay: improvement of carnitine acetyltransferase substratebinding affinity and activity in brain by feeding old rats acetyl-L-carnitine and/or R-alpha-lipoic acid. Proc. Natl. Acad. Sci. U.S.A. 99, 1876-1881.

Maher, P., and Schubert, D. (2009). The metabolic links between diabetes and Alzheimer's disease. Expert Rev. Neurother. 9, 617-630.

Marino, G., and Lopez-Otin, C. (2008). Autophagy and aging: new lessons from progeroid mice. Autophagy 4, 807-809.

Martin, B., Mattson, M. P., and Maudsley, S. (2006). Caloric restriction and intermittent feeding: two potential diets for successful brain aging. Ageing Res. Rev. 5, 332-353.

Masternak, M. M., Al-Regaiey, K. A., Bonkowski, M. S., Panici, J. A., and Bartke, A. (2005). Effect of every other day feeding diet on gene expression in normal and long-lived Ames dwarf mice. Exp. Gerontol. 40, 491-497.

Mattson, M. P., and Wan, R. (2005). Beneficial effects of intermittent feeding and caloric restriction on the cardiovascular and cerebrovascular systems. J. Nutr. Biochem. 16, 129-137.

McFarland, G.A., and Holliday, R. (1994). Retardation of senescence in cultured human diploid fibroblasts by carnosine. Exp. Cell Res. 212, 167-175.

Miller, E., Rutkowski, M., Mrowicka, M., and Matuszewski, T. (2007). Participation of reactive oxygen species in muscle damage produced by hypokinesis. Pol. Merkur. Lekarski. 22, 314-317.

Mollica, M. P., Iossa, S., Soboll, S., and Liverini, G. (2001). Acetyl-L-carnitine treatment stimulates oxygen consumption and biosynthetic function in perfused liver of young and old rats. Cell Mol. Life Sci. 59, 477-484.

Morcos, M., Du, X., Pfisterer, F., Hutter, H., Sayed, A., Thormalley, P., Ahmed, N., Baynes, J., Thorpe, S., Kukudov, G., Schlotterer, A., Bozorgmehr, F., El Baki, R. A., Stern, D., Moehrlen, F., Hamann, A., Becker, C., Zeier, M., Schwenger, V., Miftari, N., Humpert, P., Hammes, H.P., Buechler, M., Bierhaus, A., Brownlee, M., and Nawroth, P. P. (2008). Glyoxalase-1 prevents mitochondrial protein modification and enhances lifespan in C. elegans. Aging Cell 7, 260-269.

Munguia, M.E., Govezensky, T., Martinez, R., Manoutcharian, K., and Gevorkian, G. (2005). Identification of amyloidbeta 1-42 binding protein fragments by screening of a human brain cDNA library. Neurosci. Lett. 397, 79-82.

Nicolay, J. P., Schneider, J., Niemoeller, O. M., Artune, F., Portero-Otin, M., Hair, G., Jr., Thornalley, P. J., Schleicher, E., Wieder, T., and Lang, F. (2006). Stimulation of cell suicide by methylglyoxal. Cell. Physiol. Biochem. 18, 223-232.

Odashima, M., Otaka, M., Jin, M., Wada, I., Horikawa, Y., Matsuhashi, T., Ohba, R., Hatakeyama, M., Oyake, J., and Watanabe, S. (2006).Zinc-L-carnosine protects colonic mucosal injury through induction of heat shock protein 72 and suppression of NF-kB activation. Life Sci. 79, 2245-2250.

Ohkawara, T., Nishihira, J., Nagashima, R., Takeda, H., and Asaka, M. (2006). Polaprezinc protects human colon cells from oxidative injury induced by hydrogen peroxide relevant to cytoprotective heat shock proteins. World J. Gastroenterol. 12, 6178-6181.

Oppermann, U.C., Salim, S., Tjernberg, L. O., Terenius, L., and Jornvall, H. (1999). Binding of amyloid beta-peptiden to mitochondrial hydroxyacyl-CoA dehydrogenase (ERAB): regulation of an SDR enzyme activity with implications for apoptosis in Alzheimer's disease. FEBS Lett. 451, 238-242.

Orallo, F. (2008). Trans-resveratrol: a magical elixor of eternal youth? Curr. Med. Chem. 15, 1887-1898.

Orosz, F., Olah, J., and Oveadl, J. (2009). Triosephosphate isomerase deficiency: new insights into an enigmatic disease. Biochim. Biophys. Acta 1792, 1168-1174.

Pesce, V., Cormio, A., Fracasso, F., Lezze, A. M., Cantatore, P., and Gadaleta, M. N. (2002). Rat hindlimb unloadinmg: soleus and extensor digitorum longus histochemistry, mitochondrial DNA content and mitochondrial deletions. Biosci. Rep. 22, 115-125.

Poon, H. F., Calabrese, V., Calvani, M., and Butterfield, D. A. (2006). Proteomics analyses of specific protein oxidation and protein expression in aged rat brain and its modulation by L-acetylcarnitine: insights into the mechanisms of action of this proposed therapeutic agent for CNS disoders associated with oxidative stress. Antioxid. Redox Signal. 8, 381-394.

Rabbani, N., and Thornalley, P. J. (2008a). Dicarbonyls linked to damage to the powerhouse: glycation of mitochondrial proteins and oxidative stress. Biochem. Soc. Trans. 38, 1045-1050.

Rabbani, N., and Thornalley, P. J. (2008b). The dicarbonyl proteome: proteins susceptible to dicarbonyl glycation at functional sites in health, aging and disease. Ann. N. Y. Acad. Sci. 1126, 124-127.

Rhein, V., Baysang, G., Rao, S., Meir, F., Bonert, A., Muller-Spahn, F., and Eckert, A. (2009). Amyloid-beta leads to impaired cellular respiration, energy production and mitochondrial electron chain complex activities in human neuroblastoma cells. Cell. Mol. Neurobiol. 29, 1063-1071.

Rodgers, J. T., Lerin, C., Gerhart-Hines, Z., and Puigserver, P. (2008). Metabolic adaptations through PGC- $1 \alpha$ and SIRT1 pathways. FEBS Letts. 582, 46-53.

Salminen, A., and Kaamiranta, K. (2009). SIRT1: regulation of longevity via autophagy. Cell. Signal. 21, 1356-1360.

Sastre, J., Pallardo, F.V., and Vina, J. (2003). The role of mitochondrial oxidative stress in aging. Free Radic. Biol. Med. $35,1-8$.

Schlotterer, A., Kukdov, G., Bozorgmehr, F., Hutter, H., Du, X., Oikonomou, D., Ibrahim, Y., Pfisterer, F., Rabbani, N., Thormalley, P., Sayed, A., Fleming, T., Humpert, P., Schwenger, V., Zeier, M., Hamann, A., Stern, D., Brownlee, M., Bierhaus, A., Nawroth, P. P., and Morcos, M. (2009). C. elegans as model for the study of high glucose mediated lifespan reduction. Diabetes 58, 2450-2456.

Sedensky, M. M., and Morgan, P. G. (2006). Mitochondrial respiration and reactive oxygen species in mitochondrial aging mutants. Exp. Gerontol. 41, 237-245.

Sejersen, H., and Rattan, I. (2009). Dicarbonyl-induced accelerated aging in vitro in human skin fibroblasts. Biogerontology 10, 203-211.

Shao, L., Li, Q-H., and Tan, Z. M. (2004). $\mathrm{L}$-carnosine reduces telomere damage and shortening rate in cultured normal fibroblasts. Biochem. Biophys. Res. Commun. 324, 931-936.

Simonsen, A., Cumming, R. C., Brech, A., Isakson, P., Schubert, D. R., and Finley, K. D. (2008). Promoting basal levels of autophagy in the nervous system enhances longevity and oxidant resistance in adult Drosophila. Autophagy 4, 176-184.

SinhaRoy, S., Banerjee S., Ray, M., and Ray, S. (2005). Possible involvement of glutamic and/or aspartic residue(s) and requirement of mitochondrial integrity for the protective effect of creatine against inhibition of cardiac mitochondrial respiration by methyglyoxal. Mol. Cell. Biochem. 271, 167-176.

Soskic, V., Groebe, K., and Schrattenholz, A. (2007). Non-enzymic posttranslational protein modification in aging. Exp. Gerontol. 43, 247-257.

Speakman, J. R., Talbot, DA., Selman, C., Snart, S., McLaren J. S., Redman, P., Krol, E., Jackson, D. M., Johnson,. S., and Brand, M. D. (2004). Uncoupled and surviving: individual mice with high metabolism have greater mitochondrial uncoupling and live longer. Aging Cell 3, 87-95.

Sun, F., Kanthasamy, A., Anantharam, V., and Kanthasamy, A. G. (2009). Mitochondrial accumulation of polyubiquitinated proteins and differential regulation of apoptosis by polyubiquitination sites Lys-48 and 63. J. Cell Mol. Med. (in press) PMID 19432818.

Terman, A., Dalen, H., Eaton, J. W., Neuzil, J., and Brunk, U. T. (2003). Mitochondrial recycling and aging of cardiac myocytes: the role of autophagocytosis. Exp. Gerontol. 38, 863-876.

Toth, M.L., Sigmond, T., Borsos, E., Barna, J., Erdelyi, P., Takacs-Vellai, K., Orosz, L., Kovacs, A. L., Csikos, G., Sass, M., and Vellai, T. (2008). Longevity pathways converge on autophagy genes to regulate life span in Caenorhabditis elegans. Autophagy 4, 330-338.

Turk, Z. (2009). Glycotoxins, carbonyl stress and relevance to diabetes and its complications. Physiol. Res. (in press) PMID 19537931.

Van Voorhies, W. A. (2004). Live fastlive long? A commentary on a recent paper by Speakman et al. Aging Cell 3, 527-530.

Virmani, A., Gaetani, F., Imam, S., Binienda Z., and Ali, S. (2002). The protective role of L-carnitine against neurotoxicity evoked by drug of abuse, methamphetamine, could be related to mitochondrial dysfunction. Ann. N. Y. Acad. Sci. 965, 225-232.

Wada, I., Otaka, M., Jin, M., Odashima, M., Komatsu, K., Konishi, N., Matsuhashi, T., Horikawa, Y., Ohba, R., Itoh, H., and Watanabe, S. (2006). Expression of HSP72 in the gastric mucosa is regulated by gastric acid in rats - correlation of HSP72 expression with mucosal protection. Biochem. Biophys. Res. Commun. 349 , 611-618. 
Wang, H., Liu, J., and Wu, L. (2009). Methylglyoxal-induced mitochondrial dysfunction in vascular smooth muscle cells. Biochem. Pharmacol. 77, 1709-1715.

Wei, Y.-H., Wu, S.-B., Ma, Y.-S., and Lee, H.-C. (2009). Respiratory function decline and DNA mutation in mitochondria, oxidative stress and altered gene expression during aging. Chang Gung Med. J. 32, 113-132.

Westerheide, S. D., Anckar, J., Stevens, J., Sistomen, L., and Morimoto, R. I. (2009). Stress-induced regulation of the heat shock factor 1 by the deacetylase SIRT1. Science 32, 1063-1066.
Wolkow, C. A., and Iser, W. B. (2006). Uncoupling protein homologs may provide a link between mitochondria, metabolism and lifespan. Ageing Res. Rev. 5, 196-208.

Yun, C., Stanhill, A., Yang, Y., Zhang, Y. Haynes, C. M., Xu, C. F., Neubert, T. A., Mor, A., Philips, M. R., and Ron, D. (2008). Proteasomal adaptation to environmental stress links resistance to proteotoxicity with longevity in Caenorhabditis elegans. Proc. Natl. Acad. Sci. U.S.A. 105, 7094-7099.

Yuneva, M. O., Bulygina, E. R., Gallant, S. C., Kramarenko, G. G., Stvolinsky, S. L., Semyonova, M. L., and Boldyrev,
A. A. (1999). Effect of carnosine on age-induced changes in senescenceaccelerated mice. J. Anti Aging Med. 2, 337-342.

Yuneva, M. O., Kramarenko, G. G., Vetreshchak, T. V., Gallant, S. C., and Boldyrev, A. A. (2002). Effect of carnosine on Drosophila melanogaster lifespan. Bull. Exp. Biol. Med. 133 559-561.

Conflict of Interest Statement: The author declares that the research was conducted in the absence of any commercial or financial relationships that could be construed as a potential conflict of interest.
Received: 13 January 2010; paper pending published: 17 February 2010; accepted: 01 March 2010; published online: 18 March 2010.

Citation: Hipkiss AR (2010) Aging, proteotoxicity, mitochondria, glycation, $\mathrm{NAD}^{+}$and carnosine: possible interrelationships and resolution of the oxygen paradox. Front. Agi. Neurosci. 2:10. doi: 10.3389/fnagi.2010.00010

Copyright (c) 2010 Hipkiss. This is an open-access article subject to an exclusive license agreement between the authors and the Frontiers Research Foundation, which permits unrestricted use, distribution, and reproduction in any medium, provided the original authors and source are credited. 